\title{
Effect of Temperature on Growth of Vibrio paraphemolyticus and Vibrio vulnificus in Flounder, Salmon Sashimi and Oyster Meat
}

\author{
Yoo Won Kim ${ }^{1}$, Soon Ho Lee ${ }^{2}$, In Gun Hwang ${ }^{2}$ and Ki Sun Yoon ${ }^{1, *}$ \\ 1 Department of Food and Nutrition, Hoeki-dong Dongdaemun-Ku, Kyung Hee University, \\ Seoul 130-701, Korea; E-Mail: ryuwon@gmail.com \\ 2 Food Microbiology Division, National Institute of Food and Drug Safety Evaluation, Korea Food \\ and Drug Administration, Chungcheongbuk-do 363-700, Korea; \\ E-Mails: leesh13@kfda.go.kr (S.H.L.); hwangig@kfda.go.kr (I.G.H) \\ * Author to whom correspondence should be addressed; E-Mail: ksyoon@khu.ac.kr; \\ Tel.: +82-2-961-0264; Fax: +82-2-961-0261.
}

Received: 4 October 2012; in revised form: 4 December 2012 / Accepted: 10 December 2012 / Published: 13 December 2012

\begin{abstract}
Vibrio parahaemolyticus and Vibrio vulnificus are the major pathogenic Vibrio species which contaminate ready-to-eat seafood. The purpose of this study was to evaluate the risk of human illness resulting from consumption of ready-to-eat seafood such as sashimi and raw oyster meat due to the presence of $V$. parahaemolyticus and $V$. vulnificus. We compared the growth kinetics of $V$. parahaemolyticus and $V$. vulnificus strains in broth and ready-to-eat seafood, including flounder and salmon sashimi, as a function of temperature. The growth kinetics of naturally occurring $V$. vulnificus in raw oyster meat was also evaluated. The minimum growth temperatures of $V$. parahaemolyticus and $V$. vulnificus in broth were $13{ }^{\circ} \mathrm{C}$ and $11^{\circ} \mathrm{C}$, respectively. Overall, significant differences in lag time (LT) and specific growth rate (SGR) values between flounder and salmon sashimi were observed at temperatures ranging from $13{ }^{\circ} \mathrm{C}$ to $30{ }^{\circ} \mathrm{C}(p<0.05)$. The growth of naturally occurring $V$. vulnificus reached stationary phase at $\sim 4 \log \mathrm{CFU} / \mathrm{g}$ in oysters, regardless of the storage temperature. This data indicates that the population of $V$. vulnificus in oysters did not reach the maximum population density as observed in the broth, where growth of $V$. vulnificus and $V$. parahaemolyticus isolated from oysters grew up to $>8 \log \mathrm{CFU} / \mathrm{mL}$.
\end{abstract}


Keywords: $V$. parahaemolyticus; $V$. vulnificus; flounder; salmon; oyster; growth kinetics

\section{Introduction}

Vibrio parahaemolyticus and Vibrio vulnificus are the most common Vibrio species associated with illnesses resulting from consumption of raw or partially cooked seafood worldwide [1-4]. In comparison to $V$. parahaemolyticus, $V$. vulnificus is known to be more dangerous because it causes septicemia in immunocompromised individuals, and $V$. vulnificus septicemia is associated with a greater than 50\% mortality [5]. It was reported that infections caused by pathogens commonly transmitted through food have declined, or are approaching targeted national levels, with the exception of Vibrio infections [6]. In Asia, the number of reported $V$. parahaemolyticus infections has increased significantly [7]. The highest incidence of outbreaks (33) and illness (634) due to $V$. parahaemolyticus were reported between 2006 and 2010, which accounted for 17.1\% (range from 13.9\% to 20\%) of all foodborne illnesses caused by bacterial pathogens in Korea [8].

Flounder (Gwang-eo, Paralichthys spp.) and salmon (Salmonidae spp.) are the most frequently fish consumed as sashimi (raw fish) and sushi (a roll of cold rice with fish) in Asia [9]. Several cases of food poisoning due to the consumption of salmon sashimi contaminated with $V$. parahaemolyticus have been reported. Thus, it is important to control the growth of $V$. parahaemolyticus and $V$. vulnificus in sashimi and sushi, which are also becoming more popular in many other countries [10]. In addition, oysters are also frequently eaten raw and are the food most commonly associated with Vibrio infection in many countries $[11,12]$. Due to the ubiquitous nature of Vibrio species in marine environments, it is impossible to obtain seafood free of these bacteria. Therefore, it is necessary to limit the growth of Vibrio spp. in contaminated seafood in order to minimize the risk of foodborne illnesses due to seafood consumption. Unfortunately, refrigeration and freezing cannot completely destroy $V$. parahaemolyticus [13] and temperature abuse as well as mishandling of seafood at retail markets and food-service establishments may cause the rapid growth of $V$. parahaemolyticus and $V$. vulnificus $[14,15]$. Thus, it is important to understand the behavior of Vibrio spp. in raw and ready-to-eat seafood under temperature abuse conditions common during distribution and storage at retail markets.

Burnham et al. reported the differing growth and survivability of eight different $V$. parahaemolyticus and $V$. vulnificus strains in broth at a refrigeration temperature of less than $10{ }^{\circ} \mathrm{C}$ [16]. Yang et al. reported the growth and survival curves for a strain of pandemic $V$. parahaemolyticus inoculated on salmon meat over a temperature range from $0{ }^{\circ} \mathrm{C}$ to $35^{\circ} \mathrm{C}$ [17]. A few works regarding modeling of the effect of temperature on the growth of Vibrio. spp were also reported [18-21]. Miles et al. [18] modeled the effects of temperature and water activity on the growth rate of $V$. parahaemolyticus in broth. Yoon et al. [19] developed growth models for pathogenic and nonpathogenic $V$. parahaemolyticus in both broth and oyster slurry. Most recently, the effect of storage temperature (ranging from $3.6^{\circ} \mathrm{C}$ to $30.4{ }^{\circ} \mathrm{C}$ ) on $V$. paraemolyticus viability and counts of total viable bacteria in Pacific oysters was reported [20]. A predictive growth model of $V$. vulnicus in postharvest shellstock oysters were also developed and reported [21]. However, the behavior of Vibrio spp. in sashimi as a function of temperature and kind of fish has not been compared. Therefore, the primary objective of this study was to compare the growth kinetics of $V$. parahaemolyticus in flounder and salmon sashimi as a function of 
temperature, which were also compared to the growth kinetics of $V$. vulnificus and $V$. parahaemolyticus in broth. In addition, growth kinetics of naturally occurring $V$. vulnificus in oyster was evaluated in order to assess the risk involved with the consumption of raw oyster meat if oysters are not promptly refrigerated.

\section{Experimental Section}

\subsection{Bacteria Strains}

Three strains of $V$. parahaemolyticus (ATCC 17802, 27969, and 33844) and $V$. vulnificus ATCC 27562 were used. All strains were maintained at $-70{ }^{\circ} \mathrm{C}$ at a concentration of $9.5-10.0 \log \mathrm{CFU} / \mathrm{mL}$ in nutrient broth (NB; Becton Dickinson, Sparks, MD, USA) with 3\% salt that contained 20\% glycerol (Sigma Chemical Company, St. Louis, MO, USA). For each experiment, $10 \mu \mathrm{L}$ of thawed, stock culture of $V$. parahaemolyticus and $V$. vulnificus was inoculated into a $25 \mathrm{~mL}$ Erlenmeyer flask that contained $9 \mathrm{~mL}$ of sterile $\mathrm{NB}$ with $2 \%$ salt and tryptic soy broth (TSB) containing $2 \%$ salt, respectively. The flasks were then sealed with a foam plug and incubated at $36^{\circ} \mathrm{C}$ for $24 \mathrm{~h}$ at $140 \mathrm{rpm}$ on a rotary shaker (VS-8480SR, Vision Scientific, Daejun, Korea). One mL of the starter culture was then transferred into $9 \mathrm{~mL}$ of phosphate-buffered saline (PBS at $\mathrm{pH}=7.4$ ) containing $2.5 \%$ salt, which was then serially diluted prior to inoculation into both the broth and sashimi samples.

\subsection{Preparation of Inoculation in Broth and Sashimi Samples}

To compare the growth kinetics of $V$. parahaemolyticus and $V$. vulnificus in broth, $10 \mu \mathrm{L}$ of the diluted starter culture of $V$. parahaemolyticus ATCC 33844 (the fastest growing $V$. parahaemolyticus strain among all the strains tested in this study) and $V$. vulnificus ATCC 27562 were inoculated into $9 \mathrm{~mL}$ of NB containing $2 \%$ salt and TSB containing $2 \%$ salt using a sterile pipette in order to give a target population of approximately $2.5-3.5 \log \mathrm{CFU} / \mathrm{mL}$, respectively. They were then immediately incubated at $11,13,18,24,30$, and $36^{\circ} \mathrm{C}$ without agitation. At selected times post-inoculation, which were determined based on the incubation temperature of the broth, $100 \mu \mathrm{L}$ aliquots were diluted as appropriate, plated onto thiosulfate-citrate-bile salts-sucrose (TCBS; Difco, Becton Dickinson) agar in duplicate, and incubated aerobically at $36{ }^{\circ} \mathrm{C}$ for $18-20 \mathrm{~h}$. The colonies on the TCBS plates were then counted, after which, the counts from the duplicated plates were converted to log numbers. In order to compare the growth kinetics of $V$. parahaemolyticus in flounder and salmon sashimi, fresh flounder and salmon sashimi packs (150 g per pack) were purchased from a local grocery market in Seoul, Korea (Vibrio spp. were not detected in any sashimi samples used in this study). Each sample was aseptically prepared by cutting sashimi into $10 \mathrm{~g}$ pieces using the blade of a sterile knife and placed in a sterile Petri dish. Each thin $(0.2-0.3 \mathrm{~mm})$ slice of sashimi was then uniformly surface inoculated with $10 \mu \mathrm{L}$ of the diluted starter culture of $V$. parahaemolyticus using a sterile pipette to give a final concentration of $\sim 3 \log \mathrm{CFU} / \mathrm{g}$. The samples were then placed into a stomacher bag, sealed, and stored at $13,18,24,30$, and $36^{\circ} \mathrm{C}$. The minimum growth temperature of $V$. parahaemolyticus has been determined from the growth data in broth. At selected times after inoculation, sashimi samples were homogenized (Stomacher, Interscience, Paris, France) for $2 \mathrm{~min}$ in $90 \mathrm{~mL}$ of sterilized PBS containing $2.5 \%$ salt $(\mathrm{pH}=7.4)$. A $1 \mathrm{~mL}$ aliquot of diluted homogenate was spread onto TCBS in duplicate and 
incubated at $36^{\circ} \mathrm{C}$ for $24 \mathrm{~h}$. The colonies from the duplicated plates were counted and the counts were converted into log numbers. Each experiment was repeated twice with two replicates per experiment.

\subsection{Isolation of Natural V. parahaemolyticus and V. vulnificus in Shucked Oysters}

Shucked oyster (Crassostrea gigas) packs (150 g per pack) cultivated on the Southern coast of Korea were purchased from grocery markets in Seoul. The shucked oyster packs were packed in an insulated container with dry ice and transported to the laboratory within $1 \mathrm{~h}$. One pack was used for each temperature. A microbiological analysis was conducted immediately after arrival. Approximately $25 \mathrm{~g}$ of oyster meat (2-3 oysters) was added to $225 \mathrm{~mL}$ of phosphate buffered saline (PBS at $\mathrm{pH}=7.4$ ) with $2.5 \%$ salt, and the mixture was blended in a pulsifier (Microben, Bioproduct LTD, Surrey, United Kingdom) for $30 \mathrm{~s}$. The resulting homogenate was streaked onto TCBS agar and incubated for $24 \mathrm{~h}$ at $36{ }^{\circ} \mathrm{C}$. All green and yellow colonies that formed on TCBS agar were enumerated for total Vibrios. The green colonies on TCBS agar were transferred onto Chrome Vibrio agar (CV, Chromagar, Paris, France). The colonies of $V$. parahaemolyticus and $V$. vulnificus were identified as having mauve and blue colors in CV agar, respectively, and were tested again for oxidase and Gram staining. Suspicious oxidase positive and Gram-negative colonies were again confirmed as $V$. parahaemolyticus and $V$. vulnificus using an API 20NE diagnostic strip (Biomerieux, Marcy L'Etoile, France). Each isolated $V$. parahaemolyticus and $V$. vulnificus strain was maintained at $-70{ }^{\circ} \mathrm{C}$ in $\mathrm{NB}$ with $2 \%$ salt and TSB containing $2 \%$ salt, respectively.

\subsection{Determination of Growth Kinetics of V. vulnificus in Shucked Oyster Meat}

From September to March in 2010-2011, only V. vulnifius was consistently detected in shucked oysters obtained from seafood markets. Nor $V$. parahaemolyticus and $V$. vulnificus were detected at all samples from December to February in 2010-2011. Thus, only the growth kinetics of natural $V$. vulnificus in oyster meat was evaluated in this study. Shucked oyster meat ( $25 \mathrm{~g}$ portions) purchased from a grocery market were placed in a stomacher bag using sterilized tweezers and sealed (under micro-aerobic conditions). Each sample was stored at temperatures of $16,18,24,30$, and $36{ }^{\circ} \mathrm{C}$ for the growth of naturally occurring $V$. vulnificus in oyster meat. At selected times during storage, samples were diluted into $75 \mathrm{~mL}$ with phosphate buffered saline (PBS) with $2.5 \%$ salt and homogenized in a pulsifier (Microben) for 30 seconds. The homogenates were serially diluted in sterile PBS with $2.5 \%$ salt solution and spread onto Chrome Vibrio (CV) agar, in duplicate and incubated $37{ }^{\circ} \mathrm{C}$ for $24 \mathrm{~h}$. The colonies of $V$. vulnificus from duplicated plates were counted and the counts were converted into log numbers.

\subsection{Effect of Temperature on Growth Kinetics of V. parahaemolyticus and V. vulnificus.}

Growth kinetics of $V$. parahaemolyticus and $V$. vulnificus in broth, sashimi and oyster meat were compared by the construction of growth curves. The growth kinetics parameters including lag time (LT) and specific growth rate (SGR) at each temperature were determined by the modified Gompertz equation using GraphPad Prism 4.0 (GraphPad Software, San Diego, CA, USA) [19]. The equation used was as follows:

$$
\mathrm{Y}=\mathrm{N}_{0}+\mathrm{C} \times \exp (-\exp ((2.718 \times \mathrm{SGR} / \mathrm{C}) \times(\mathrm{Lag}-\mathrm{t})+1))
$$


In this equation, $\mathrm{Y}$ is the viable cell count $(\log \mathrm{CFU} / \mathrm{g}), \mathrm{N}_{0}$ is the initial $\log$ number of cells, $\mathrm{C}$ is the difference between the initial and final cell numbers, SGR is the maximum specific growth rate (log $\mathrm{CFU} / \mathrm{h}$ ), $\mathrm{LT}$ is the lag time before growth and $\mathrm{t}$ is the sampling time.

For the effect of temperature on the values of LT and SGR, the Davey equation [22] and square root equation [23] were used, respectively. The Davey equation for LT was used as follows:

$$
\mathrm{Y}=\mathrm{a}+(\mathrm{b} / \mathrm{T})+\left(\mathrm{c} / \mathrm{T}^{2}\right)
$$

where $\mathrm{Y}$ is LT (day), a, b, and c are regression coefficients without biological meaning, and $\mathrm{T}$ is temperature. The square-root equation for SGR was used as follows:

$$
\mathrm{Y}=\{\mathrm{b}(\mathrm{T}-\mathrm{Tmin})\}^{2}
$$

where $\mathrm{Y}$ is the growth rate, $b$ is the regression coefficient, and Tmin is the theoretical minimum temperature required for the growth of the organism to occur. The goodness-of-fit of the data was evaluated based on the coefficient of determination $\left(\mathrm{R}^{2}\right)$, which was provided by GraphPad Prism.

\subsection{Statistical Analysis}

The experiment was repeated twice with two replicates per experiment. The obtained results were analyzed using the Statistical Analysis System, SAS V 9.1 (SAS Institute Inc., Cary, NC, USA). The significant differences among the groups were determined by analysis of variance (ANOVA) and the means were also separated using t-tests $(p<0.05)$.

\section{Results and Discussion}

\subsection{Comparison of Growth Kinetics of V. parahaemolyticus and V. vulnificus in Broth}

In this study, we used the fastest growing $V$. parahaemolyticus strain 33844 among all the strains tested in this study (data not shown) and compared it to the growth kinetics for $V$. vulnificus strain 27562 in broth at $11,1318,24,30$, and $36{ }^{\circ} \mathrm{C}$ (Table 1). The growth curves in broth were fitted well to a Gompertz equation with a high degree regarding goodness-of-fit $\left(\mathrm{R}^{2}=0.995\right.$ to 997$)$. Lag time (LT), specific growth rate (SGR), and maximum population density (MPD) were compared between $V$. parahaemolyticus and $V$. vulnificus as a function of temperature. Significant differences $(p<0.05)$ in the values of LT, SGR and MPD between $V$. parahaemolyticus and $V$. vulnificus were also observed at all temperatures. In addition, higher SGR of $V$. vulnificus was observed in comparison to that of $V$. parahaemolyticus. The average MPD values of $V$. parahaemolyticus and $V$. vulnificus were 9.37 and 8.90, respectively. Although $V$. parahaemolyticus strain 33844 (purchased from ATCC) was the fastest growing strain among the tested strains in our study, its growth was much slower than that of the pathogenic strain JL 223 isolated from 72 Korean raw oysters used in a previous study [19]. At $20{ }^{\circ} \mathrm{C}$, a longer LT value $(10.32 \mathrm{~h}$ ) for $V$. parahaemolyticus strain 33844 in broth was predicted in this study, compared to LT values for pathogenic $(8.16 \mathrm{~h})$ and nonpathogenic $(6.25 \mathrm{~h}) \mathrm{V}$. parahaemolyticus isolated from raw oysters. 
Table 1. Effect of temperature on Lag time (LT) and specific growth rate (SGR), and maximum population density (MPD) of $V$. parahaemolyticus and $V$. vulnificus in broth.

\begin{tabular}{|c|c|c|c|c|c|c|c|}
\hline & Strain & $11^{\circ} \mathrm{C}$ & $13^{\circ} \mathrm{C}$ & $18^{\circ} \mathrm{C}$ & $24^{\circ} \mathrm{C}$ & $30^{\circ} \mathrm{C}$ & $36^{\circ} \mathrm{C}$ \\
\hline \multirow{3}{*}{ LT (h) } & $V$. parahaemolyticus & - & $48.64 \pm 5.23^{\mathrm{A}}$ & $16.94 \pm 2.33^{\text {B }}$ & $2.52 \pm 0.16^{\mathrm{C}}$ & $2.22 \pm 0.33^{\mathrm{C}}$ & $1.61 \pm 0.87^{\mathrm{C} . \mathrm{D}}$ \\
\hline & & & & & & & \\
\hline & V. vulnificus & $127.80 \pm 4.97^{\mathrm{A}}$ & - & $10.49 \pm 3.17^{\mathrm{B}}$ & $4.60 \pm 1.09^{\mathrm{C}}$ & $2.40 \pm 0.72^{\mathrm{D}}$ & $1.46 \pm 0.23^{\mathrm{E}}$ \\
\hline \multirow{2}{*}{$\operatorname{SGR}(\log / \mathrm{h})$} & $V$. parahaemolyticus & - & $0.035 \pm 0.04^{\mathrm{E}}$ & $0.160 \pm 0.14^{\mathrm{D}}$ & $0.561 \pm 0.02^{\mathrm{C}}$ & $0.847 \pm 0.04^{\mathrm{B}}$ & $1.184 \pm 0.35^{\mathrm{A}}$ \\
\hline & V. vulnificus & $0.027 \pm 0.08^{\mathrm{D}}$ & - & $0.365 \pm 0.21^{\mathrm{B}}$ & $0.687 \pm 0.22^{\mathrm{C}}$ & $1.029 \pm 0.31^{\mathrm{A}}$ & $1.096 \pm 0.28^{\mathrm{A}}$ \\
\hline \multirow{2}{*}{ MPD (log) } & $V$. parahaemolyticus & & $9.48 \pm 0.11^{\mathrm{A}}$ & $9.66 \pm 0.25^{\mathrm{A}}$ & $9.12 \pm 0.23^{\text {A.B }}$ & $8.86 \pm 1.03^{\mathrm{B}}$ & $9.74 \pm 0.62^{\mathrm{A}}$ \\
\hline & V. vulnificus & $7.54 \pm 1.34^{\mathrm{C}}$ & - & $8.69 \pm 0.77^{\mathrm{B}}$ & $9.66 \pm 0.29^{\mathrm{A}}$ & $9.62 \pm 0.32^{\mathrm{A}}$ & $9.00 \pm 0.85^{\mathrm{A}, \mathrm{B}}$ \\
\hline
\end{tabular}

Capital letters (A, B, C, D, E) mean ( $\mathrm{n}=4)$ within a row with different superscripts are significantly different at the $p<0.05$ level. 
Compared to the SGR values of pathogenic (0.42 $\log \mathrm{CFU} / \mathrm{h})$ and nonpathogenic $V$. parahaemolyticus (0.42 $\log \mathrm{CFU} / \mathrm{mL})$, a lower SGR value $(0.30 \log \mathrm{CFU} / \mathrm{h})$ was also predicted for $V$. parahaemolyticus strain 33844. This indicates that care must be given when selecting a strain to develop a growth predictive model, and various strains isolated from seafood must be compared for their growth kinetics under various environmental conditions. These results again confirm the results regarding the study conducted by Burnham et al. [16], where strain to strain differences in the growth and survival of various $V$. parahaemolyticus and $V$. vurnificus were observed at refrigeration temperatures.

The effect of temperature on the values of LT and SGR were determined and modeled (Table 2). The Davey equation for LT and the square root equation for SGR were used for the data collected in the broth. The minimum growth temperatures of $V$. parahaemolyticus and $V$. vulnificus in broth were $13{ }^{\circ} \mathrm{C}$ and $11{ }^{\circ} \mathrm{C}$, respectively. In contrast to the results of this study, the minimum growth temperature of $V$. parahemolyticus in broth has been reported to be $5^{\circ} \mathrm{C}$ and $8.3^{\circ} \mathrm{C}$ [18]. Yoon et al. [19] also reported that pathogenic and nonpathogenic $V$. parahaemolyticus grew at $10{ }^{\circ} \mathrm{C}$ in broth. Burnham et al. [16] observed that five of eight $V$. parahaemolyticus strains and seven of eight $V$. vulnificus strains had increases in viable counts during 10 days of storage at $8{ }^{\circ} \mathrm{C}$ and $10{ }^{\circ} \mathrm{C}$, respectively, with significant differences in growth between the various strains.

\subsection{Comparison of Growth Kinetics of V . parahaemolyticus in Flounder and Salmon Sashimi}

The results of the present monitoring study showed that no contamination of $V$. parahaemolyticus was found in flounder and salmon sashimi from three different grocery markets in Korea. The growth curves for $V$. parahaemolyticus in flounder and salmon sashimi also fitted well the modified Gompertz equation, with a high degree of goodness-of-fit $\left(R^{2}=0.9394\right.$ to 0.9948$)$. The values of LT, SGR, and MPD were compared between flounder and salmon sashimi (Table 3). The minimum growth temperature of $V$. parahaemolyticus in flounder and salmon sashimi was found to be $13{ }^{\circ} \mathrm{C}$, as observed in the broth and the average MPD was $~ 6.96 \mathrm{log} \mathrm{CFU} / \mathrm{g}$, regardless of the storage temperature. Significant differences in LT values were observed in the sashimi sample and broth at all temperatures, but no significant differences in SGR values were observed between the sashimi sample and broth at $13{ }^{\circ} \mathrm{C}(p<0.05)$. When MPD on flounder and salmon were compared to that on broth, they were significantly different at all temperatures $(p<0.05)$. In a comparison of growth kinetics on salmon sashimi, the LT values of $V$. parahaemolyticus on flounder were significantly shorter $(p<0.05)$, regardless of storage temperature. However, no significant differences in the values of SGR between flounder and salmon sashimi were observed at temperatures $<24{ }^{\circ} \mathrm{C}$ (Table 3). Overall, the growth of $V$. parahaemolyticus in broth was more rapid compared to the growth on flounder and salmon sashimi (Table 3), indicating that the predicted growth model of $V$. parahaemolyticus, which were developed in broth, may not be appropriate for the risk assessment of $V$. parahaemolyticus in the sashimi sample. Moreover, more rapid growth of $V$. parahaemolyticus in flounder sashimi was observed compared to salmon sashimi. Our results demonstrate that behavior of $V$. parahaemolyticus may vary depending on the kind of fish (Figure 1). 
Table 2. Model for lag time (LT) and specific growth rate (SGR) of V. parahaemolyticus and V. vulnificus in broth, sashimi, and oyster.

\begin{tabular}{|c|c|c|c|c|}
\hline Model & Parameter & Strain & $\mathbf{R}^{2}$ & Equation \\
\hline \multirow{4}{*}{ Broth } & \multirow{2}{*}{ LT } & V. parahaemolyticus & 0.997 & $\mathrm{Y} *=19.31+(-1227 / \mathrm{T})+\left(20945 / \mathrm{T}^{2}\right)$ \\
\hline & & V. vulnificus & 0.997 & $\mathrm{Y}=2.784+(-65.53 / \mathrm{T})+\left(387.6 / \mathrm{T}^{2}\right)$ \\
\hline & \multirow{2}{*}{ SGR } & V. parahaemolyticus & 0.976 & $\mathrm{Y}=\{0.03489(\mathrm{~T}-4.213)\}^{2}$ \\
\hline & & V. vulnificus & 0.996 & $\mathrm{Y}=\{0.03115(\mathrm{~T}+1.316)\}^{2}$ \\
\hline \multirow{4}{*}{$\begin{array}{c}\text { Flounder } \\
\text { sashimi } \\
\text { Salmon } \\
\text { sashimi }\end{array}$} & LT & $V \cdot$ parahaemolyticus & 0.992 & $\mathrm{Y}^{\dagger}=-28.04+(-1037 / \mathrm{T})+\left(-1.867 / \mathrm{T}^{2}\right)$ \\
\hline & SGR & $V$. parahaemolyticus & 0.990 & $\mathrm{Y}=\{0.02017(\mathrm{~T}-3.223)\}^{2}$ \\
\hline & $\mathrm{LT}$ & V. parahaemolyticus & 0.993 & $\mathrm{Y}=-25.43+(993.2 / \mathrm{T})+\left(1792 / \mathrm{T}^{2}\right)$ \\
\hline & SGR & V. parahaemolyticus & 0.939 & $\mathrm{Y}=\{0.01052(\mathrm{~T}+13.52)\}^{2}$ \\
\hline \multirow{2}{*}{ oyster } & $\mathrm{LT}$ & V. vulnificus & 0.950 & $\mathrm{Y}=24.80+(-1211 / \mathrm{T})+\left(17449 / \mathrm{T}^{2}\right)$ \\
\hline & SGR & V. vulnificus & 0.930 & $\mathrm{Y}=\{0.01380(\mathrm{~T}+5.604)\}^{2}$ \\
\hline
\end{tabular}

$* \mathrm{LT}=\mathrm{a}+(\mathrm{b} / \mathrm{T})+\left(\mathrm{c} / \mathrm{T}^{2}\right) ;{ }^{\dagger} \mathrm{Y}=\{\mathrm{b}(\mathrm{T}-\mathrm{Tmin})\}^{2}$.

Table 3. Effect of temperature on Lag time (LT) and specific growth rate (SGR) of $V$. parahaemolyticus in flounder and salmon sashimi and broth.

\begin{tabular}{|c|c|c|c|c|c|c|}
\hline & Sample & $13^{\circ} \mathrm{C}$ & $18^{\circ} \mathrm{C}$ & $24^{\circ} \mathrm{C}$ & $30^{\circ} \mathrm{C}$ & $36^{\circ} \mathrm{C}$ \\
\hline \multirow{3}{*}{$\mathrm{LT}(\mathrm{h})$} & Flouder & ${ }^{\mathrm{A}} 51.35 \pm 1.78^{\mathrm{a}}$ & ${ }^{\mathrm{A}} 30.96 \pm 3.66^{\mathrm{b}}$ & $\mathrm{A}^{\mathrm{A}} 14.79 \pm 2.99^{\mathrm{c}}$ & ${ }^{\mathrm{A}} 3.90 \pm 1.22^{\mathrm{d}}$ & ${ }^{\mathrm{A}} 2.77 \pm 1.07^{\mathrm{d}}$ \\
\hline & Salmon & ${ }^{\mathrm{B}} 61.16 \pm 3.11^{\mathrm{a}}$ & ${ }^{\mathrm{B}} 36.81 \pm 1.87^{\mathrm{b}}$ & ${ }^{\mathrm{B}} 18.54 \pm 3.03^{\mathrm{c}}$ & ${ }^{\mathrm{B}} 6.98 \pm 3.17^{\mathrm{d}}$ & ${ }^{\mathrm{B}} 5.64 \pm 2.12^{\mathrm{d}}$ \\
\hline & broth & ${ }^{\mathrm{C}} 48.64 \pm 4.18^{\mathrm{a}}$ & ${ }^{\mathrm{C}} 16.94 \pm 5.15^{\mathrm{b}}$ & ${ }^{\mathrm{C}} 2.52 \pm 4.81^{\mathrm{c}}$ & ${ }^{\mathrm{C}} 2.22 \pm 1.18^{\mathrm{c}}$ & ${ }^{\mathrm{C}} 1.61 \pm 0.96^{\mathrm{c}, \mathrm{d}}$ \\
\hline \multirow{3}{*}{ SGR(log/h) } & Flouder & ${ }^{\mathrm{A}} 0.037 \pm 0.04^{\mathrm{d}}$ & ${ }^{\mathrm{A}} 0.105 \pm 0.09^{\mathrm{c}}$ & ${ }^{\mathrm{A}} 0.152 \pm 0.21^{\mathrm{c}}$ & ${ }^{\mathrm{A}} 0.304 \pm 0.02^{\mathrm{b}}$ & ${ }^{\mathrm{A}} 0.435 \pm 0.21^{\mathrm{a}}$ \\
\hline & Salmon & ${ }^{A} 0.059 \pm 0.02^{d}$ & ${ }^{\mathrm{A}} 0.103 \pm 0.12^{\mathrm{c}}$ & ${ }^{\mathrm{A}} 0.185 \pm 0.27^{\mathrm{b}}$ & ${ }^{\mathrm{B}} 0.219 \pm 0.76^{\mathrm{a}, \mathrm{b}}$ & ${ }^{\mathrm{B}} 0.256 \pm 0.24^{\mathrm{a}}$ \\
\hline & broth & ${ }^{\mathrm{A}} 0.035 \pm 0.03^{\mathrm{e}}$ & ${ }^{\mathrm{B}} 0.160 \pm 0.19^{\mathrm{d}}$ & ${ }^{\mathrm{B}} 0.561 \pm 0.35^{\mathrm{c}}$ & ${ }^{\mathrm{C}} 0.847 \pm 0.55^{\mathrm{b}}$ & ${ }^{\mathrm{C}} 1.184 \pm 0.59^{\mathrm{a}}$ \\
\hline \multirow{3}{*}{ MPD $(\log )$} & Flouder & ${ }^{\mathrm{A}} 6.53 \pm 0.67^{\mathrm{b}}$ & $\mathrm{A}^{\mathrm{A}} 7.30 \pm 1.21^{\mathrm{a}}$ & $\mathrm{A}^{\mathrm{A}} 7.21 \pm 1.09^{\mathrm{a}}$ & $\mathrm{A}^{\mathrm{A}} 7.60 \pm 0.89^{\mathrm{a}}$ & $\mathrm{A}^{\mathrm{A}} 7.46 \pm 0.45^{\mathrm{a}}$ \\
\hline & Salmon & ${ }^{\mathrm{B}} 5.54 \pm 1.65^{\mathrm{c}}$ & ${ }^{\mathrm{B}} 6.51 \pm 0.53^{\mathrm{b}}$ & ${ }^{\mathrm{B}} 6.45 \pm 0.65^{\mathrm{b}}$ & ${ }^{\mathrm{A}} 7.34 \pm 0.43^{\mathrm{a}}$ & ${ }^{\mathrm{B}} 6.95 \pm 0.66^{\mathrm{b}}$ \\
\hline & Broth & ${ }^{\mathrm{C}} 9.48 \pm 0.87^{\mathrm{a}}$ & ${ }^{\mathrm{C}} 9.66 \pm 1.07^{\mathrm{a}}$ & ${ }^{\mathrm{C}} 9.12 \pm 1.19^{\mathrm{a}, \mathrm{b}}$ & ${ }^{\mathrm{B}} 8.86 \pm 0.53^{\mathrm{b}}$ & ${ }^{\mathrm{C}} 9.74 \pm 0.32^{\mathrm{a}}$ \\
\hline
\end{tabular}

Small letters mean $(\mathrm{a}, \mathrm{b}, \mathrm{c}, \mathrm{d})(\mathrm{n}=4)$ within a row with different superscripts are significantly different at the $p<0.05$ level.

Capital letters mean (A, B, C) $(\mathrm{n}=4)$ within a column with different superscripts are significantly different at the $p<0.05$ level. 
Figure 1. Effect of temperature on the values of Lag time (LT) and Specific growth rate (SGR) of $V$. parahaemolyticus in broth, flounder, and salmon sashimi. Data $(\mathrm{n}=4)$ for LT and SGR were fitted using Equations (2) and (3), respectively.
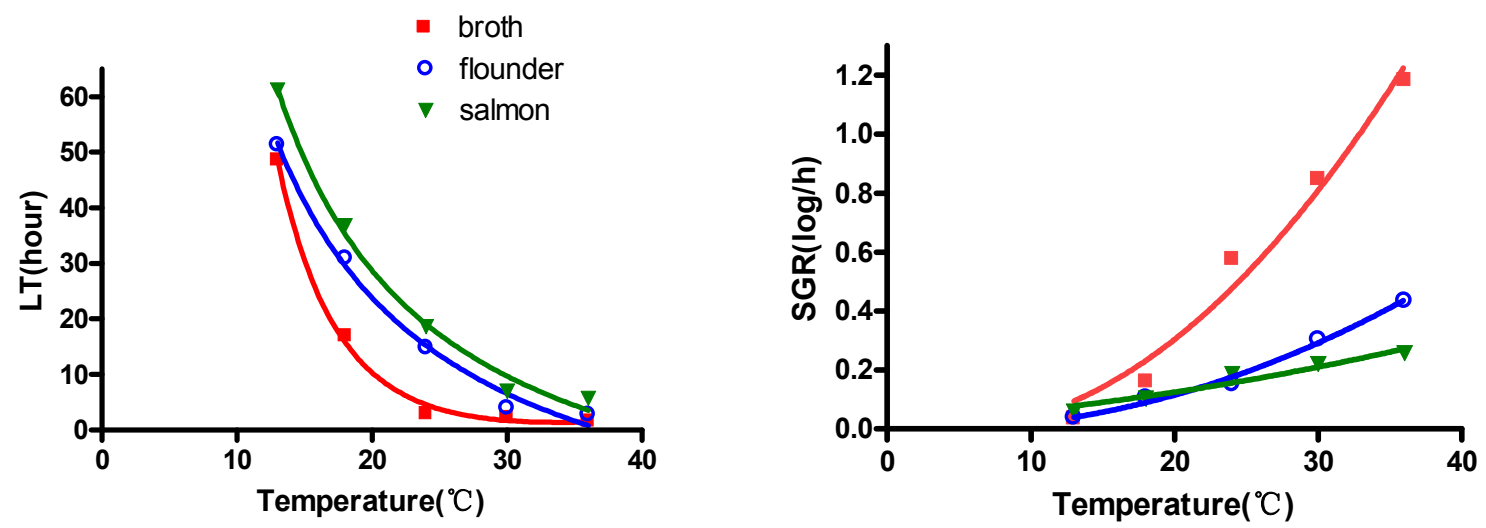

Compared to the SGR values of pathogenic $(0.42 \log \mathrm{CFU} / \mathrm{h})$ and nonpathogenic $V$. parahaemolyticus $(0.42 \log \mathrm{CFU} / \mathrm{mL})$, a lower SGR value $(0.30 \log \mathrm{CFU} / \mathrm{h})$ was also predicted for $V$. parahaemolyticus strain 33844 . This indicates that care must be given when selecting a strain to develop a growth predictive model, and various strains isolated from seafood must be compared for their growth kinetics under various environmental conditions. These results again confirm the results regarding the study conducted by Burnham et al. [16], where strain to strain differences in the growth and survival of various $V$. parahaemolyticus and $V$. vurnificus were observed at refrigeration temperatures.

The effect of temperature on the values of LT and SGR were determined and modeled (Table 2). The Davey equation for LT and the square root equation for SGR were used for the data collected in the broth. The minimum growth temperatures of $V$. parahaemolyticus and $V$. vulnificus in broth were $13{ }^{\circ} \mathrm{C}$ and $11^{\circ} \mathrm{C}$, respectively. In contrast to the results of this study, the minimum growth temperature of $V$. parahemolyticus in broth has been reported to be $5{ }^{\circ} \mathrm{C}$ and $8.3{ }^{\circ} \mathrm{C}$ [18]. Yoon et al. [19] also reported that pathogenic and nonpathogenic $V$. parahaemolyticus grew at $10{ }^{\circ} \mathrm{C}$ in broth. Burnham et al. [16] observed that five of eight $V$. parahaemolyticus strains and seven of eight V. vulnificus strains had increases in viable counts during 10 days of storage at $8{ }^{\circ} \mathrm{C}$ and $10{ }^{\circ} \mathrm{C}$, respectively, with significant differences in growth between the various strains.

The effect of temperature on LT and SGR in broth, sashimi, and oyster was also modeled (Table 2). The Davey equation was used in order to evaluate the LT of $V$. parahaemolyticus, respectively at 13,18 , 24,30 , and $36{ }^{\circ} \mathrm{C}$, where growth was possible. A square root equation was used to determine the relationship between SGR and the storage temperature of $V$. parahaemolyticus on flounder, salmon, and broth in this work. In comparison to this study, Yang et al. [17] determined the growth and survival curves for a strain of pandemic $V$. parahaemolyticus $\mathrm{Tgq}+01$ (serotype O3:K6) on salmon meat within the temperature range of $0{ }^{\circ} \mathrm{C}$ to $35^{\circ} \mathrm{C}$. They used salmon, which was soaked in sterile water containing $100 \mathrm{ppm}$ chlorine. The minimum growth temperature of $V$. parahaemolyticus on salmon meat in their study was $12.1^{\circ} \mathrm{C}$, while the minimum growth temperature of $V$. parahaemolyticus on salmon sashimi was $13{ }^{\circ} \mathrm{C}$ in this study. At $30{ }^{\circ} \mathrm{C}$, a 2-fold longer LT (6.98 h) was observed in our study compared to an LT value of $3.43 \mathrm{~h}$ in their study. Furthermore, the value of SGR for $V$. parahaemolyticus in salmon meat being twice as rapid compared to that of the $V$. parahaemolyticus in salmon sashimi $(0.558 \log \mathrm{CFU} / \mathrm{h}$ 
vs. $0.219 \log \mathrm{CFU} / \mathrm{h}$ ), indicated more rapid growth of $V$. parahaemolyticus Tgq+01(serotype O3:K6) on salmon meat than the $V$. parahaemolyticus ATCC strain in salmon sashimi.

\subsection{Growth Kinetics of Natural V. vulnificus in Shucked Oyster Meat}

In the present study, we observed the growth of natural $V$. vulnificus in shucked oyster meat, which was purchased from a local grocery market. Since no growth of natural $V$. vulnificus was observed in oyster meat at a temperature lower than $16{ }^{\circ} \mathrm{C}$, growth kinetics of naturally occurring $V$. vulfnicius in oyster meat was determined at $16,18,24,30$, and $36^{\circ} \mathrm{C}$ (Figure 2). Recently, a predictive growth model for $V$. parahaemolyticus in Pacific oysters was developed [20], where 0.030 and $0.282 \log _{10} \mathrm{CFU} / \mathrm{h}$ of growth rate was observed at 18.4 and $30.4{ }^{\circ} \mathrm{C}$, respectively. On the other hand, the specific growth rate of $V$. vulnificus in oyster meat in our study was $0.080 \log _{10} \mathrm{CFU} / \mathrm{h}$ at $18{ }^{\circ} \mathrm{C}$ and $0.328 \log _{10} \mathrm{CFU} / \mathrm{h}$ at $30{ }^{\circ} \mathrm{C}$ (Figure 2). Although a direct comparison between their study and our study may not be appropriate, the growth of $V$. vulnificus was much faster than that of $V$. parahaemolyticus in oyster meat. In addition, DaSilva et al. [21] developed a predictive model for $V$. vulnficus in Eastern shellstock oysters (Crassostrea Virginica) over a temperature range of 5 to $30{ }^{\circ} \mathrm{C}$. The estimated average growth rates at $15,20,25$, and $30{ }^{\circ} \mathrm{C}$ were $0.022,0.042,0.087$, and $0.093 \log \mathrm{MPN} / \mathrm{h}$, respectively. Overall, much lower growth rates of $V$. vulnificus were observed in shellstock oysters compared to our study, where we used shucked oyster meat.

Figure 2. Growth curves of natural $V$. vulnificus in oyster as a function of time at various temperatures. Data $(n=4)$ were fitted using Equation (1). LT: lag time, SGR: specific growth rate symbols.
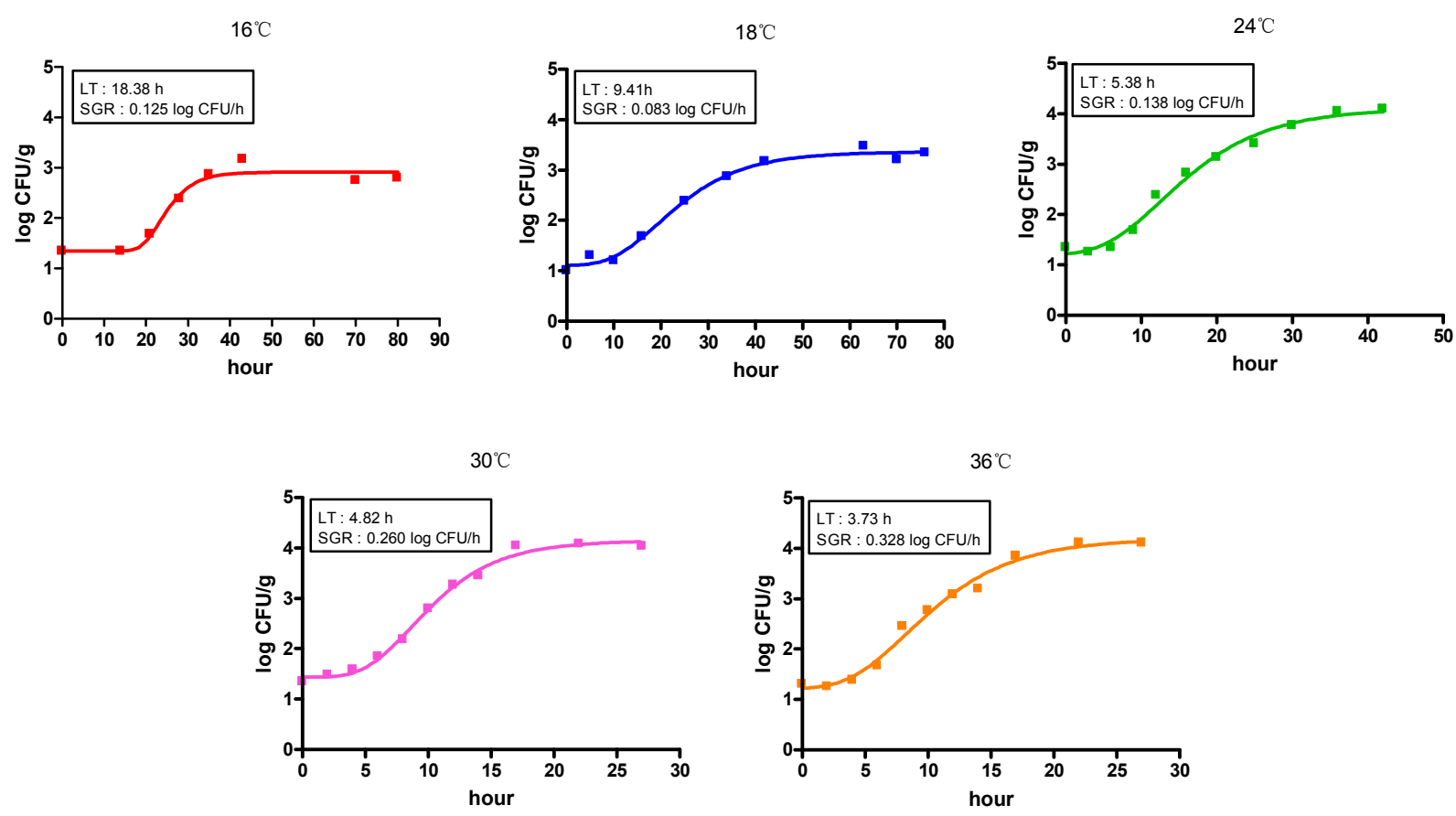

In addition, the minimum growth temperature of $V$. vulnificus in shellstock oysters was $15^{\circ} \mathrm{C}$ in their work. In this study, the minimum growth temperature of natural $V$. vulnificus in oyster meat purchased 
from a local market was $16^{\circ} \mathrm{C}$ (Figure 3), indicating that the growth of $V$. vulnificus may not be a major risk at retail markets as long as the shucked oyster pack is kept refrigerated.

Figure 3. Effect of temperature on the values of lag time (LT) and specific growth rate (SGR) of natural $V$. vulnificus in oysters. Data $(\mathrm{n}=4)$ for LT and SGR were fitted using Equations (2) and (3), respectively.
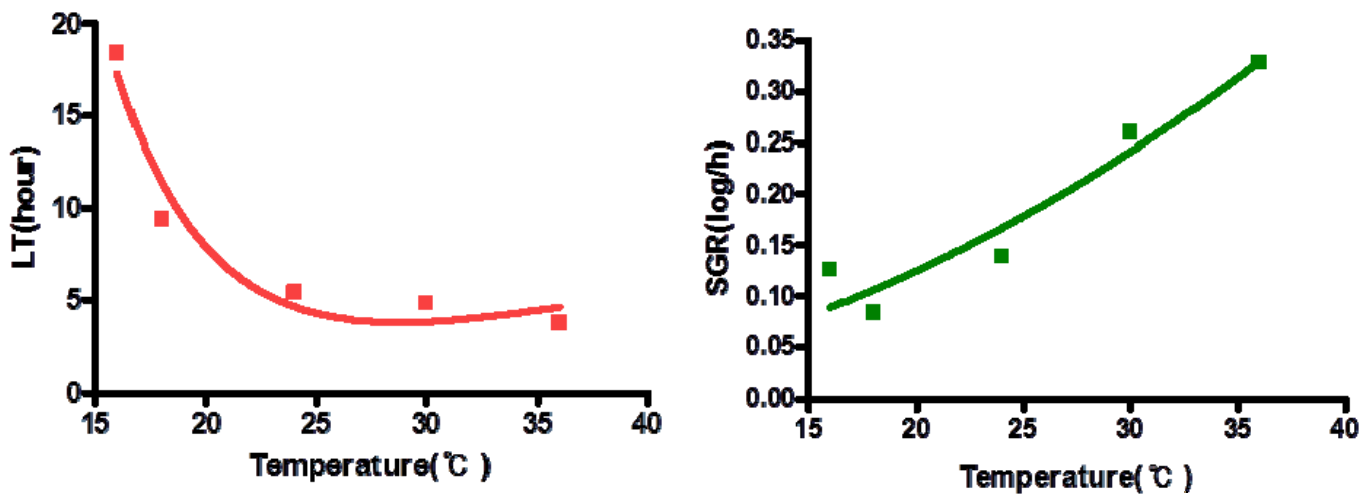

As shown in Table 4, the MPD of natural $V$. vulnificus in oysters reached a peak of $\sim 4 \log \mathrm{CFU} / \mathrm{g}$ from an initial contamination level of 1-1.3 $\log \mathrm{CFU} / \mathrm{g}$, regardless of storage temperature, while MPD of the inoculated $V$. vulnificus strain 27562 in broth was over $9 \log \mathrm{CFU} / \mathrm{mL}$ from an initial contamination level of $2.5 \log \mathrm{CFU} / \mathrm{mL}$.

Table 4. Comparisons of maximum population density (MPD) of natural V. vulnificus in oyster and inoculated $V$. vulnificus (ATCC 27562) in broth.

\begin{tabular}{|c|c|c|c|c|c|c|}
\hline Sample & & $16^{\circ} \mathrm{C}$ & $18^{\circ} \mathrm{C}$ & $24^{\circ} \mathrm{C}$ & $30{ }^{\circ} \mathrm{C}$ & $36^{\circ} \mathrm{C}$ \\
\hline \multirow{2}{*}{ Broth } & Initial density * & - & $2.44 \pm 0.91$ & $3.33 \pm 0.44$ & $3.32 \pm 0.29$ & $2.75 \pm 0.45$ \\
\hline & MPD & - & $8.70 \pm 1.09$ & $9.10 \pm 0.45$ & $8.90 \pm 0.34$ & $9.00 \pm 0.89$ \\
\hline \multirow{2}{*}{ Oyster } & Initial density $^{\dagger}$ & $1.34 \pm 0.22$ & $1.00 \pm 0.32$ & $1.31 \pm 0.46$ & $1.30 \pm 0.23$ & $1.34 \pm 0.17$ \\
\hline & MPD & $2.79 \pm 0.58$ & $3.47 \pm 0.12$ & $4.13 \pm 0.54$ & $4.08 \pm 0.52$ & $4.10 \pm 0.36$ \\
\hline
\end{tabular}

* Initial inoculation level; ${ }^{\dagger}$ Initial contamination level $(n=4)$ in oyster.

These results indicate that the populations of naturally occurring $V$. vulnificus in oysters did not reach the maximum population density observed in the broth. However, the infectious dose of V. vulnifcus for the high risk group is $2 \log \mathrm{CFU} / \mathrm{g}$, therefore, for the protection of consumers careful storage and consumption guidelines for oysters at retail markets and restaurants must be emphasized. In this study, only $V$. vulnifius was consistently detected in oysters during the period of September to March and the average population of $V$. vulnifius in shucked oyster meat at the retail grocery market was $1-1.3 \log \mathrm{CFU} / \mathrm{g}$.

Lee et al. [24] also reported $V$. parahaemolyticus as the dominant Vibrio in retail oysters during August to September. In their study, the total Vibrio number was $\sim 4 \log$ MPN/g and the average number of $V$. parahaemolyticus in Korean retail oysters was $\sim 3 \log \mathrm{MPN} / \mathrm{g}$. This discrepancy might between 
these two studies be due to the seasonal differences during the sampling period as well as the different purchase sites of the oyster samples.

Although it was not consistent, we were able to isolate both $V$. parahaemolyticus and $V$. vulnificus from shucked oyster meat purchased from a grocery market in Korea. Thus, these organisms were inoculated into broth and stored at $11{ }^{\circ} \mathrm{C}$ (Figure 4), which was the minimum growth temperature of natural $V$. parahaemolyticus and $V$. vulnificus in broth. As previously mentioned, the minimum growth temperature for strains $V$. parahaemolyticus 33844 and $V$. vulnificus 27562 purchased from ATCC were $13{ }^{\circ} \mathrm{C}$ and $11^{\circ} \mathrm{C}$ in broth, respectively (Table 1). Again, this result indicates that the behaviors of naturally occurring Vibrio strains in oysters are different from the behavior of the reference ATCC strain. As shown in Figure 4, V. vulnificus ATCC strain 27562 grew more slowly than $V$. parahaemolyticus and V. vulnificus isolated from retail oysters, indicating that growth models of Vibrio. spp. in the literature should be validated with natural $V$. parahaemolyticus and $V$. vulnificus in oysters.

Figure 4. Comparison the growth curves of inoculated $V$. vulnificus and $V$. parahaemolyticus isolated from oyster and V. vulnificus ATCC 27562 in broth at $11^{\circ} \mathrm{C}$. Data $(\mathrm{n}=4)$ were fitted using Equation (1).

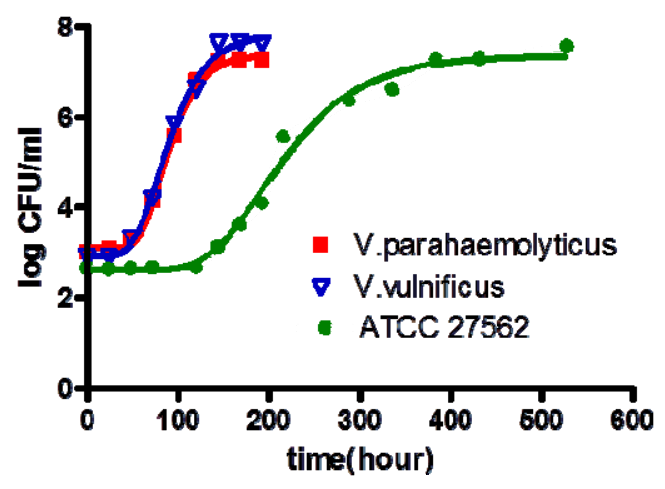

\section{Conclusions}

$V$. vulnificus was more frequently detected than $V$. parahaemolyticus in oysters from grocery markets in Korea from September to March. In this study, natural $V$. vulnificus in oysters grew to $\sim 4 \log$ CFU/g, regardless of the storage temperature tested. However, when V. vulnificus strain 27562 and naturally occurring $V$. vulnificus isolated from retail oysters were inoculated in broth, they grew to $\sim 9 \log \mathrm{CFU} / \mathrm{mL}$ in broth, regardless of storage temperature. These results indicate that the populations of $V$. vulnificus in oysters did not reach the maximum population density observed in the broth. In addition, natural $V$. parahaemolyticus and $V$. vulnificus were isolated from retail oysters and their growth curves were compared to those of $V$. vulnificus ATCC strain 27562. Their minimum growth temperature was $11{ }^{\circ} \mathrm{C}$ in broth, while the minimum growth temperature regarding $V$. parahaemolyticus strain 33844 was $13{ }^{\circ} \mathrm{C}$ in broth and sashimi. These results indicate that storage of ready to eat sashimi and oysters at temperatures $<10^{\circ} \mathrm{C}$ at retail markets will control the growth of Vibrio spp. and thus minimize the risk of illness associated with Vibrios due to raw and ready-to-eat seafood consumption. Therefore, strict temperature guidelines during the transportation and selling of raw and ready-to-eat seafood must be implemented at retail markets. The present data will be used to develop the tertiary growth model for $V$. parahaemolyticus and $V$. vulnificus from harvest to the point of sale in order to manage the risk of 
illness as a result of sashimi and raw oyster consumption. However, more extensive research for evaluating the growth behaviors of $V$. parahaemolyticus and $V$. vulnificus strains in various seafoods is needed for an overall risk assessment of raw seafood consumption.

\section{Acknowledgments}

This study was supported by the Korea Food Drug Administration Research Grant (10162-055).

\section{Conflict of Interest}

The authors declare no conflict of interest.

\section{References}

1. Su, Y.C.; Liu, C. Vibrio parahaemolyticus: A concern of seafood safety. Food Microbiol. 2007, 24, $549-558$.

2. Harth, E.; Matsuda, L.; Hernandez, C.; Rioseco, M.L.; Romero, J.; Gonzalez-Escalona, N.; Martinez-Urtaza, J.; Espejo, R.T. Epidemiology of Vibrio parahaemolyticus outbreaks, southern Chile. Emerg. Infect. Dis. 2009, 15, 163-168.

3. Centers for Disease Control and Prevention (CDC). Preliminary Food Net Data on The Incidence of Infection with Pathogens Transmitted Commonly Through Food-10 States, 2009. Available online: www.cdc.gov/mmwr/preview/mmwrhtml/mm5914a2.htm (accessed on 8 May 2012).

4. Chang, H.C.; Chen, M.L.; Su, Y.C.; Pai, J.Y.; Chiu, T.H. Molecular characterizations of pathogenic Vibrio parahaemolyticus isolated from Southern Taiwan oyster-growing environment. Food Control. 2011, 22, 245-251.

5. Kumamoto, K.S.; Vukich, D.J. Clinical infections of Vibrio vulnificus: A case report and review of the literature. J. Emerg. Med. 1998, 16, 61-66.

6. Centers for Disease Control and Prevention (CDC). Preliminary Food Net Data on The Incidence of Infection with Pathogens Transmitted Commonly Through Food-10 States, 2005. Available online: www.cdc.gov/mmwr/preview/mmwrhtml/mm5514a2.htm (accessed on 14 September 2011).

7. Liu, F.; Guan, W.; Alam, M.J.; Shen, Z.; Zhang, S.; Li, L.; Shinoda, S.; Shi, L. Pulsed-field gel electrophoresis typing of multidrug-resistant vibrio parahaemolyticus isolated from various sources of seafood. J. Health Sci. 2009, 55, 783-789.

8. Korea Food and Drug Administration (KFDA). The Report of Foodborne Illness in Korea. Available online: www.kfda.go.kr (in Korean) (accessed on 11 December 2011).

9. Ministry of Health \& Welfare. Korea National Health and Nutrition Examination Survey (KNHNES). Available online: knhanes.cdc.go.kr (in Korean) (accessed on 5 July 2010).

10. Hamada-Sato, N.; Usui, K.; Kobayashi, T.; Imada, C.; Watanabe, E. Quality assurance of raw fish based on HACCP concept. Food Control 2005, 16, 301-307.

11. Centers for Disease Control and Prevention (CDC). Vibrio Vulnificus Infections Associated with Raw Oyster Consumption-Florida, 1981-1992. Available online: www.cdc.gov/mmwr/preview/ mmwrhtml/00020736.htm (accessed on 23 October 2011). 
12. Hlady, W.G. Vibrio Infections Associated with Raw Oyster Consumption in Florida, 1981-1994. J. Food Protect. 1997, 60, 353-357.

13. Vasudevan, P.; Marek, P.; Daigle, S.; Hoagland, T.; Venkitanarayanan, K.S. Effect of chilling and freezing on survival of vibrio parahaemolyticus on fish fillets. J. Food Safety 2002, 22, 209-218.

14. Gooch, J.A.; DePaola, A.; Bowers, J.; Marshall, D.L. Growth and survival of vibrio parahaemolyticus in postharvest American oysters. J. Food Protect. 2002, 65, 970-974.

15. Food and Drug Administration (FDA). Quantitative Risk Assessment on The Public Health Impact of Pathogenic Vibrio Parahaemolyticus Strains in Raw Oysters. Available online: www.fda.gov/Food/ScienceResearch/ResearchAreas/RiskAssessmentSafetyAssessment/ucm0504 21.htm (accessed on 19 July 2005).

16. Burnham, V.E.; Janes, M.E.; Jakus, L.A.; Supan, J.; DePaola, A.; Bell, J. Growth and survival differences of Vibrio vulnificus and Vibrio parahaemolyticus strains during cold storage. J. Food Sci. 2009, 74, 314-318.

17. Yang, Z.Q.; Jiao, X.A.; Li, P.; Pan, Z.M.; Huang, J.L.; Gu, R.X.; Fang, W.M.; Chao, G.X. Predictive model of Vibrio parahaemolyticus growth and survival on salmon meat as a function of temperature. Food Microbiol. 2009, 26, 606-614.

18. Miles, D.W.; Ross, T.; Olley, J.; McMeekin, T.A. Development and evaluation of a predictive model for the effect of temperature and water activity on the growth rate of Vibrio parahaemolyticus. Int. J. Food Microbiol. 1997, 38, 133-142.

19. Yoon, K.S.; Min, K.J.; Jung, Y.J.; Kwon, K.Y.; Lee, J.K.; Oh, S.W. A model of the effect of temperature on the growth of pathogenic and nonpathogenic Vibrio parahaemolyticus isolated from oysters in Korea. Food Microbiol. 2008, 25, 635-641.

20. Fernandez-Piquer, J.; Bowman, J.P.; Ross, T.; Tamplin, M.L. Predictive models for the effect of storage temperature on vibrio parahaemolyticus viability and counts of total viable bacteria in pacific oysters (crassostrea gigas). Appl. Environ. Microb. 2011, 77, 8687-8695.

21. DaSilva, L.; Parveen, S.; DePaola, A.; Bowers, J.; Brohawn, K.; Tamplin, M.L. Development and validation of a predictive model for the growth of vibrio vulnificus in postharvest shellstock oysters. Appl. Environ. Microb. 2012, 78, 1675-1681.

22. Lee, J.K.; Jung, D.W.; Eom, S.Y.; Oh, S.W.; Kim, Y.; Kwak, H.S.; Kim, Y.H. Occurrence of Vibrio parahaemolyticus in oysters from Korean retail outlets. Food Control. 2008, 19, 990-994.

23. Davey, K.R. A predictive model for combined temperature and water activity on microbial growth during the growth phase. J. Appl. Microbiol. 1989, 67, 483-488.

24. Ratkowsky, D.A.; Olley, J.; McMeekin, T.A.; Ball, A. Relationship between temperature and growth rate of bacterial cultures. J. Bacteriol. 1982, 149, 1-5.

(C) 2012 by the authors; licensee MDPI, Basel, Switzerland. This article is an open access article distributed under the terms and conditions of the Creative Commons Attribution license (http://creativecommons.org/licenses/by/3.0/). 